\title{
Analysis of Chronic Skin Diseases using Artificial Neural Network
}

\author{
Sudhakar Singh \\ Department of Electrical Engineering, SoE \\ Gautam Buddha University, Greater Noida, India - \\ 201312
}

\author{
Shabana Urooj \\ Department of Electrical Engineering, SoE \\ Gautam Buddha University, Greater Noida, India - \\ 201312
}

\begin{abstract}
This paper presents a novel method of skin diseases classification. The complete work is divided into four parts. First is preprocess the image then segment the image by using modified sobel edge detection technique, and extract the features of the segmented image, extracted features are sub divided in to sub space features and calssified the features by artificial Neural Network(ANN). The performance of the different training algorithm has been investigated. Mean Square Error (MSE) is evaluated. Bayesian regularization backpropagation algorithm gives minimum MSE is 4.8561e13 and gradient is $1.6337 \mathrm{e}-08$ at 190 epochs. LevenbergMarquardt backpropagation algorithm provides MSE $1.0559 \mathrm{e}-$ 10 and gradient is $9.9001 \mathrm{e}-08$ at 105 epochs. Resilient backpropagation algorithm $3.5354 \mathrm{e}-07$ and gradient is $8.5468 \mathrm{e}-06$ at 347 epochs. Scaled conjugate gradient backpropagation algorithm give MSE 0.02269 and gradient is $8.6124 \mathrm{e}-07$ at 115 epochs.
\end{abstract}

\section{Keywords}

Image segmentation, Feature extraction, Feature selection, ANN, Classification

\section{INTRODUCTION}

Skin cancer is one of the disease in the modern world which causes death to quite a few number of patients. Medical professionals and researchers are trying to find the root cause of the disease and produce effective medicines for the same. Most of the patients don't know about their skin cancer till it is in final stages. The basic diagnosis of a skin cancer is done using visual inspection by a general physician and if any further investigation needed the case is referred to a dermatologist. A biopsy and pathological analysis is carried out to know the type of cancer (Basal Cell Skin Cancer (BCC), Squamous-Cell Skin Carcinoma and Malignant Melanoma). The deadliest among these three are Malignant Melanoma. It has an asymmetrical area, color variation, irregular border and diameter is often greater than $6 \mathrm{~mm}$. Malignant Melanoma can cause death if it goes undetected or it spreads in the human skin.

Skin illnesses like tanning, pigment darkening, sunburn, skin cancers, and infective diseases are growing at a more rapidly due to litter, ultraviolet light, and global warming. Pollution increases the risk of Eczema and Psoriasis being the major contributors in the skin disease. A one percent decline in ozone leads to a 2-5\% boost in the prevalence of skin cancers and other related disease. Computer aided detection (CAD) can be used on the skin disease images to assist the skin expert radiologist as a subsequent reader. CAD can find and differentiate the skin disease that a skin disease expert may not sprout.
Once the CAD analysis has been done, the skin expert can take the decision based upon the classification results. Dermoscopy is a first and foremost extremely helpful standard technique for diagnosing the spiteful cells of skin disease [1]. The vital signs of dermoscopy are broadening in correctness matched with naked-eye examination (up to $28 \%$ in the case of sensitivity and up to $14 \%$ in the case of specificity), thereby sinking the occurrence of pointless surgical removals of benign lesions [2-3]. Skin infection is a large amount added unsafe if it is not found in the premature stages [4].

\subsection{Related Work}

A number of the fuzzy logic techniques examined in preceding research used for color and furthermore skin assessment, including color histogram examination for color marking and skin laceration intolerance [5]. Unfairness, constraint recognition is also defined in skin lesion using fuzzy clustering and texture analysis [6]. The fuzzy clustering segmentation technique is used for the adaptive removing background of skin color for part segmentation [7] and fuzzy c-means clustering considered for pelt scratch analysis [8]. Adaptive fuzzy, c-means improved technique uses local spatial continuity for cluster pattern evaluation [9], here skin area segmented by fuzzy estimation grade modeling [10]. Scheduled starting the foundation of artificial intelligence, soft computing, and image processing, used for design as of a number of authors have furthermore, the addition of area understanding concerning the individuality of objects increases the segmentation precision [11], [12]. The fuzzy rules are functional among every single point of thoughtfulness (applicant points used for shape detection) single individuals substantial the rule sets are reserved for investigation, by applying a number of criteria of decimation. Based deceitful on the segmented regions of interest, the morphological features and spatial distribution are constructed. These features are classified by a multi-class support vector machine classifier [13].Melanoma parameters including Asymmetry, Border, color, diam, (ABCD) it checked by the lesion Image analysis tools.

\section{MATERIAL AND METHODS}

\subsection{Sobel Operators}

Very first step in image investigation is preprocess and then segment the image. It is units an image into its component or matters. The subdivision is carried depends on the difficult being observed. Certain it's essential to segment the object from the background to read the image properly and classify the content of the image for this reason.

There are two methods of segmentation one is discontinuity recognition technique and Likeness detection technique. In the first technique, one approach is to partition an image based on abrupt changes in gray-level image. The second technique is 
based on the threshold and region growing. This paper discuss the first techniques using Edge Detection method.

By using the Sobel operators the partial derivation in gradient may be approximated in skin images which are shown in the masks below [14]:

\begin{tabular}{|l|l|l|}
\hline 1 & 2 & 1 \\
\hline 0 & 0 & 0 \\
\hline-1 & -2 & -1 \\
\hline
\end{tabular}

\begin{tabular}{|l|l|l|}
\hline-1 & 0 & 1 \\
\hline-2 & 0 & 2 \\
\hline-1 & 0 & 1 \\
\hline
\end{tabular}

Fig.1: The

\section{Sobel masks}

The gradient of an image $f(x, y)$ at position $(x, y)$ is given as:

$$
\nabla f=\frac{G_{x}}{G_{y}}=\frac{\partial f / \partial x}{\partial f / \partial y}
$$

The magnitude of this gradient vector is given below the:

$$
|\nabla f|=\sqrt{G_{x}^{2} G_{y}^{2}}
$$

The gradient takings it's extreme rate of rise of $\mathrm{f}(\mathrm{x}, \mathrm{y})$ per unit distance in the path of $\nabla f$. The gradient measure is usually estimated by:

$$
\alpha(x, y)=\tan ^{-1}\left(G_{x} / G_{y}\right)
$$

These two masks collected with any of the equations are castoff to obtain the gradient size of the image from the original image.

\subsection{Feature Extraction}

Feature selection eliminates the dimensionality of data by selecting only a subset of deliberate features (analyst variables) to generate a model. Series criterion frequently involves the minimization of a accurate compute of extrapolative fault for models fit to dissimilar subsets. Feature selection is preferable to feature conversion when the unique units and meaning of features are important and the modeling goal is to identify an influential subset. When categorical features are present, and arithmetical transformations are unfortunate, feature selection becomes the principal means of dimension diminution.

\subsection{Color features}

Color features is one of the significant features of images. Color features are defined subject to a particular color space or model. Different color spaces have been used such as RGB, LUV, HSV and HMMD [15]. A numeral of significant color features have been estimated in the research papers, including color histogram, color moments(CM) [16], color coherence vector (CCV) [17] and color correlogram [16], etc. Amongst them, CM is one of the simplest yet very operative feature. Very common moments are mean, standard deviation and sleekness, the analogous calculation can be described as follows:

$$
\begin{aligned}
& \mu_{i}=\frac{1}{n} \sum_{j=1}^{n} X_{i j} \\
& \sigma_{i}=\sqrt{\frac{1}{n} \sum_{i=1}^{n}\left(X_{i j}-\mu_{i}\right)^{2}} \\
& \gamma_{i}=\sqrt[3]{\frac{1}{n} \sum_{i=1}^{n}\left(X_{i j}-\mu_{i}\right)^{2}}
\end{aligned}
$$

where $X_{i j}$ represnts color value of the $\mathrm{i}$-th color component of the $\mathrm{j}$-th image pixel and $\mathrm{n}$ is the total number of pixels in the image. $\mu_{i}, \sigma_{i}, \gamma_{i}(\mathrm{i}=1,2,3)$ represent the mean, standard deviation and skewness of every channel of an image correspondingly. DCD, CSD and SCD symbolize the main color descriptor, color building descriptor and scalable color descriptor separately [18].

\subsection{Texture Feature}

Here some classical statistical texture measures [19]: entropy, energy, contrast, and homogeneity, which are derived from a grey level co-occurrence matrix (GLCM). For texture analysis two-dimensional gray-level co-occurrence matrix is used. In order to find the locative dependence of brightness (graylevel) values, which helps to find valuable information about the neighboring pixels in an image. The definition of cooccurrence matrix $\mathrm{p}$ of an image I of size $\mathrm{N} * \mathrm{~N}$ is given below. Here, the distance between the interested pixel and its neighbor is defined as the offset $(\Delta x, \Delta y)$. Then, we can extract four different features from GLCM. The feature of Entropy, Energy, Contrast and Homogeneity are given below:

$$
\begin{aligned}
& \text { Entropy }=-\sum_{m} \sum_{n} P^{2}[m, n] \log P[m, n] \\
& \text { Energy }=\sum_{m} \sum_{n} P^{2}[m, n] \\
& \text { Co }=\sum_{m} \sum_{n}(m-n)^{2} c(m-n)
\end{aligned}
$$

Homogeneity

$$
H o=\sum_{m} \sum_{n} c \frac{(m, n)}{1+(m-n)^{2}}
$$

Gray-Level Run-Number Vector: represents the sum distribution of the number of runs with gray level $i$.

$$
P_{g}(i)=\sum_{j=1}^{R l} P(m, n)
$$




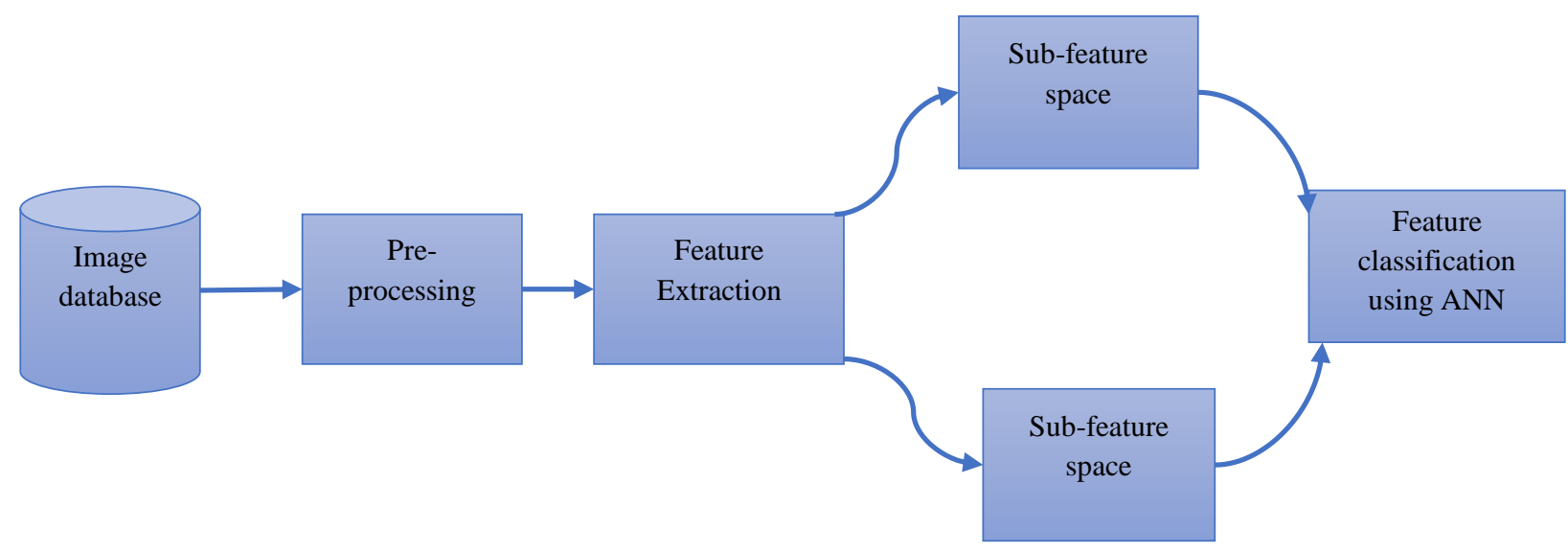

Fig2. : Proposed technique for melanoma classification

\section{CLASSIFICATION}

\section{ANN model for melanoma image}

\section{classification}

Some papers used NN classifier [20], SVM classifier [21] or AdaBoost classifier [22] for image classification. The goal of this work is to apply Artificial Neural Network (ANN) for image classification. After the images were preprocessed and extracted features, they would present in the large representation space. Thus, they would be projected into the Sub-space in order to analysis easily and reduce dimensions of image's feature.

This feature vector is the input of ANN for image classification based on a sub-space. Every ANN has 3 layers: input, hidden and output. The number nodes of input layer are equal to the dimension of feature vector. The number nodes of output are equal to $n$, the number of classes. In our proposal model, there are some parameters as the following:

$\mathrm{k}$ : the number of sub-space

$\mathrm{n}$ : the number of classes

\section{RESULT AND DISCUSSION}

Fig.3 (a) shows the test image of different kind of skin diseases, (b) shows the mask of the test image, (c) border removed of the test image, (d) final binary mask of the test image, (e) final segmented image, (f) image quality in terms of metric values of segmented image. Table 1 presents selected features of the above skin images.

In this paper six different skin problem based images have been taken. All six images has been segmented and calculated metric value using proposed technique. All test images have been segmented and found that the images having metric value greater than 60 . Images having metric value more than 60 are abnormal. From table1 melanocytic image having lowest metric value and malignant melanoma having highest metric value. Total ten features are estimated in which four selected features are shown in fig.4 (a). From fig.4 (a) it is clear that malignant image having highest metric value, standard deviation, mean value, and diameter. From fig. 3 and fig.4 it is observed that the mole image having lowest metric value, lowest standard deviation, lowest mean value and having larger diameter than BCC image. From fig.4 (b) bcc and mole images having maximum and minimum threshold

value respectively. Maximum value of roundness is depicted in fig.4 (b) for melanoma images and minimum value of roundness for the malignant image. Sleekness value of melanoma image is highest and lowest for mole image.

Fig.5 shows the training performance of the of the selected feature. The best performance is achieved, $9.8093 \mathrm{e}-08$ at epochs 305. Fig.6 presents confusion matrix of different skin images. From the confusion matrix performance of classification is observed. From fig.7 validation of performance is checked and observed gradient is $6.3719 \mathrm{e}-07$ at epoch 305.Fig. 8 presents error histogram for the 20 bins. Table2 represents performance of the different algorithms for the image classification. From table2 Bayesian regularization backpropagation training algorithm is better than other three algorithms. Levenberg-Marquardt backpropagation training algorithm is also better algorithm in terms of epochs and MSE for image classification, which takes less iterations to get minimum error as compare to other two algorithms (Resilient backpropagation and Scaled conjugate gradient backpropagation).

\section{CONCLUSION}

In this paper total work is accomplished in four steps. First image is preprocessed and segmented. Segmentation of skin images have been done using modified sobel edge detection technique. Extract features from segmented image and selected the required features. Then these selected features are divided into small $\mathrm{n}$ sub feature space, these small sub feature space is applied for classification using ANN. Four different training algorithms have been applied for the investigation of the performance. Bayesian regularization backpropagation training algorithm is better than other three algorithms it takes 190 epochs for minimum MSE 4.8561e-13 and gradient 1.6337e-08. Performance of Scaled conjugate gradient backpropagation algorithm was lowest in terms of MSE 0.02269 and gradient $8.6124 \mathrm{e}-07$ at 115 epochs. 
(a)

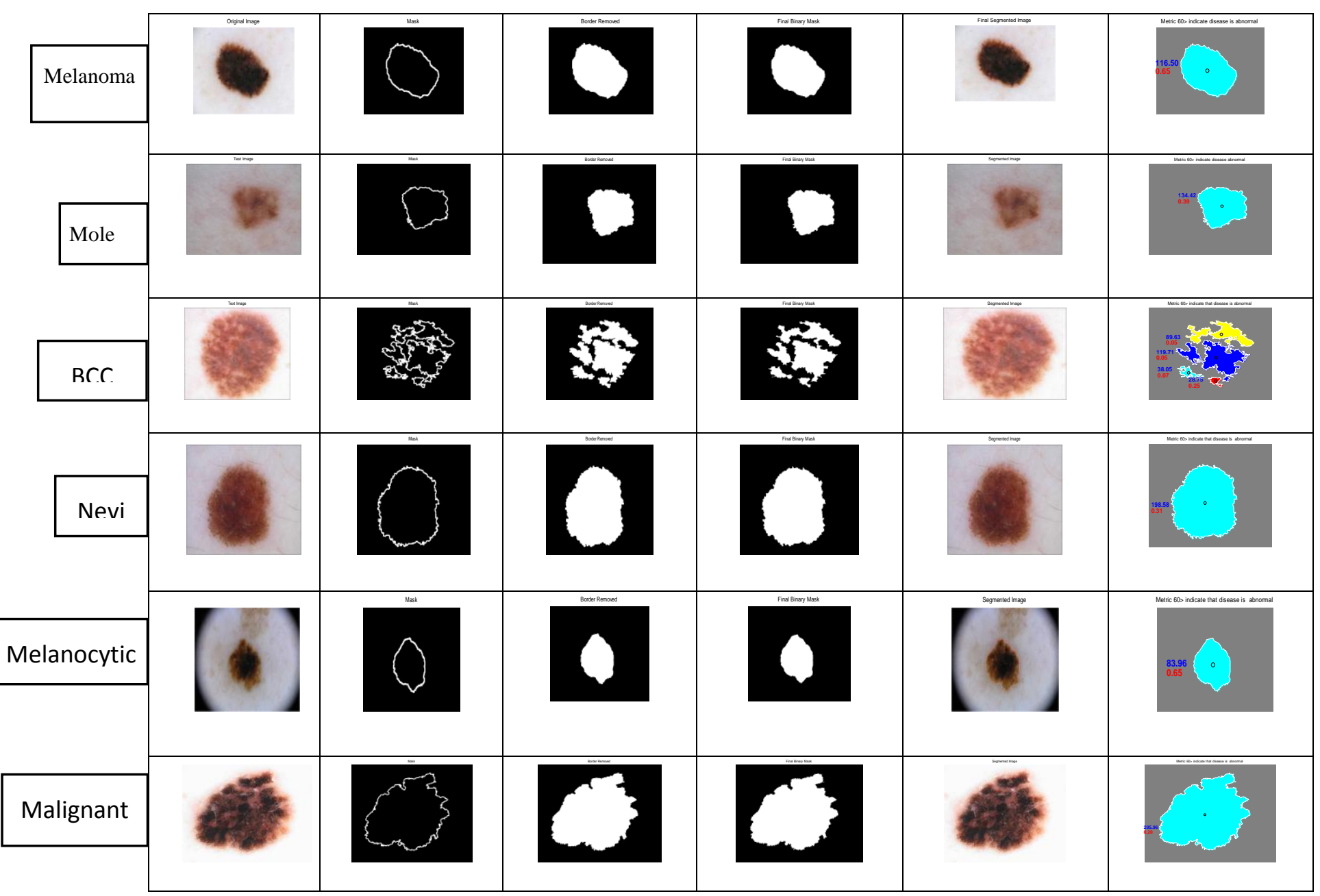

Fig.3: (a) test image, (b) mask, (c) border removed, (d) final binary mask, (e)segmented image, (f) metric image

Table:1 Features of the Test Images

\begin{tabular}{|c|c|c|c|c|c|c|c|c|c|}
\hline Threshod & Roundness & $\begin{array}{l}\text { Diamete } \\
\mathbf{r}\end{array}$ & Centroids & Mean & Moment3 & $\begin{array}{l}\text { Max. } \\
\text { Skewnes } \\
\text { s }\end{array}$ & $\begin{array}{l}\text { Max. } \\
\text { Kurtosis }\end{array}$ & S.D. & $\begin{array}{l}\text { Metric } \\
\text { value }\end{array}$ \\
\hline 0.1255 & 0.6507 & 116.4966 & $\begin{array}{l}122.0482, \\
96.8557\end{array}$ & 76.3333 & $\begin{array}{l}1.0429 \mathrm{e}+ \\
06\end{array}$ & 2.3601 & 6.7828 & $\begin{array}{l}109.572 \\
9\end{array}$ & 116.50 \\
\hline 0.0660 & 0.3925 & 134.4240 & $\begin{array}{l}2.2512 \mathrm{e}+02 \\
1.3280 \mathrm{e}+02\end{array}$ & 56.5556 & $\begin{array}{l}3.6301 \mathrm{e}+ \\
05\end{array}$ & 1.6292 & 4.4559 & 80.0043 & 134.42 \\
\hline 0.2241 & 0.2480 & 28.7460 & $\begin{array}{l}2.0566 \mathrm{e}+02 \\
2.2642 \mathrm{e}+02\end{array}$ & 66.1120 & $\begin{array}{l}7.1723 \mathrm{e}+ \\
05\end{array}$ & 1.6386 & 4.3031 & 95.2596 & 119.32 \\
\hline 0.1279 & 0.3138 & 198.5787 & $\begin{array}{l}1.7341 \mathrm{e}+02 \\
1.6323 \mathrm{e}+02\end{array}$ & 59.0000 & 419398 & 2.1392 & 6.0934 & 83.5823 & 198.58 \\
\hline 0.1050 & 0.6492 & 83.9563 & $\begin{array}{l}1.7341 \mathrm{e}+02, \\
1.6323 \mathrm{e}+02\end{array}$ & 52.4444 & $\begin{array}{l}3.3151 \mathrm{e}+ \\
05\end{array}$ & 2.3018 & 6.6031 & 75.1228 & 83.96 \\
\hline 0.1284 & 0.2003 & 295.9603 & $\begin{array}{l}2.3630 \mathrm{e}+02, \\
1.9757 \mathrm{e}+02\end{array}$ & 83.6667 & $\begin{array}{l}1.1713 \mathrm{e}+ \\
06\end{array}$ & 2.3501 & 6.7520 & 118.32 & 295.96 \\
\hline
\end{tabular}

\footnotetext{
S.D.=standard deviation
} 


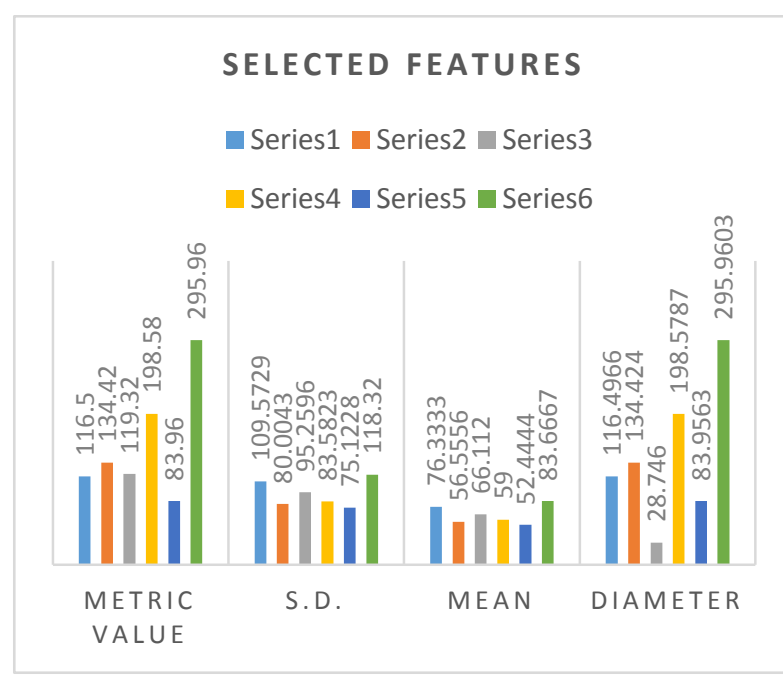

Fig .4(a): Extracted Features

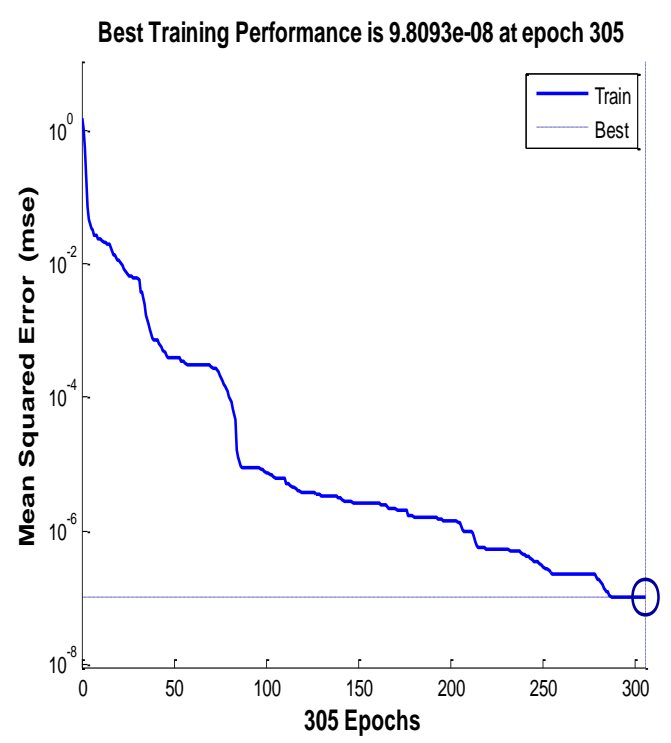

Fig.5: Training performance
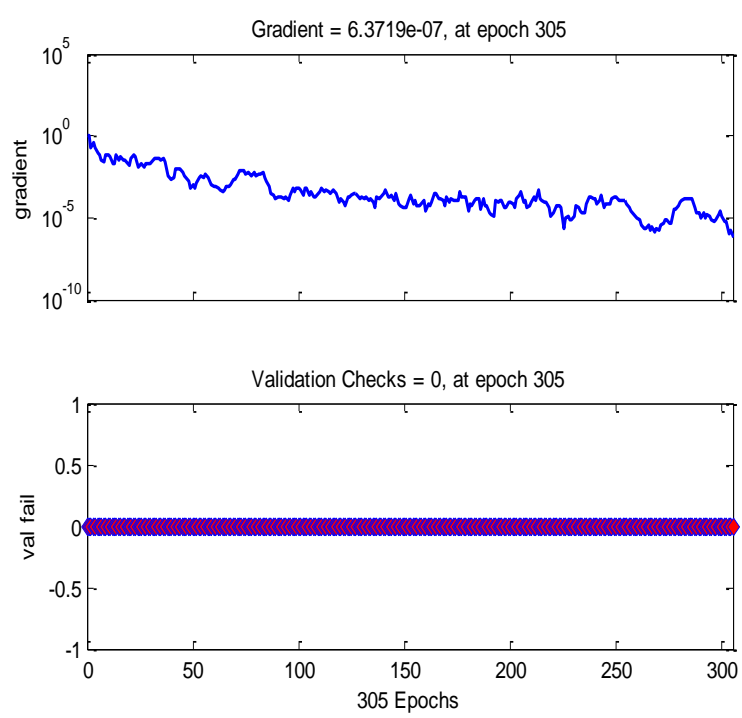

Fig.7: Validation performance

\section{SELECTED FEATURES}

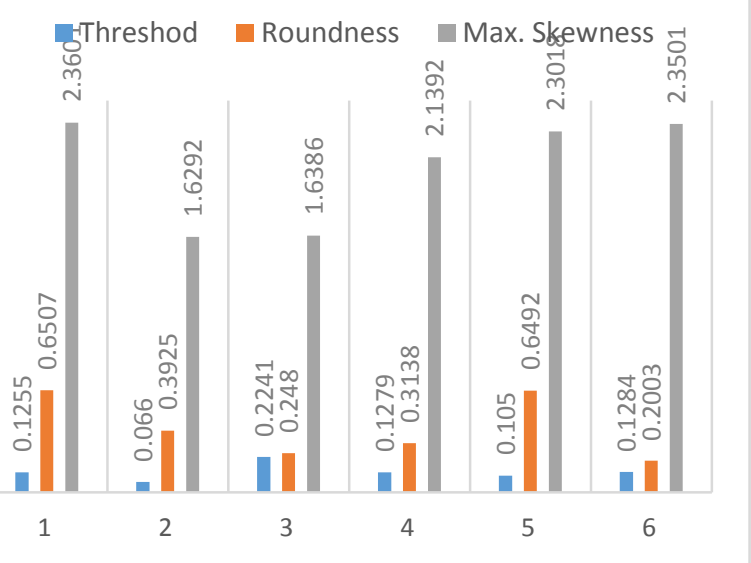

Fig.4 (b): Extracted Features

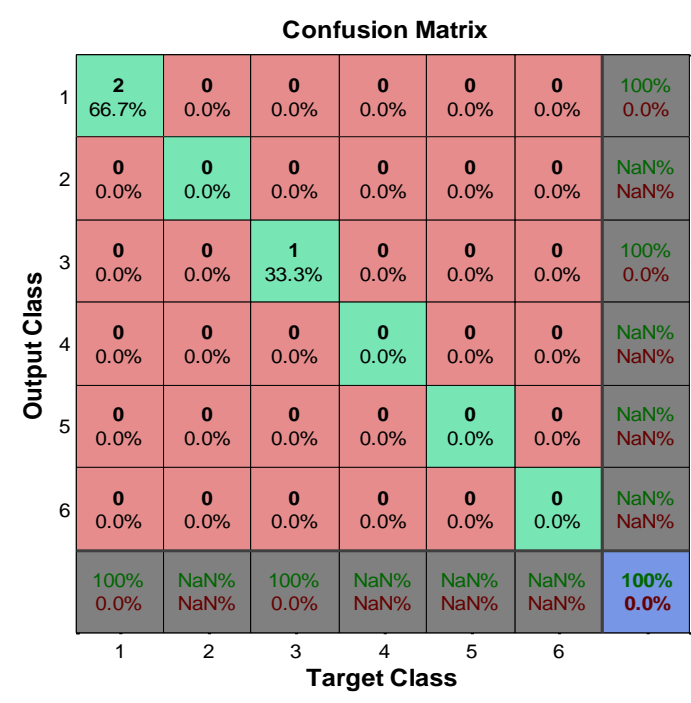

Fig.6: Confusion matrix

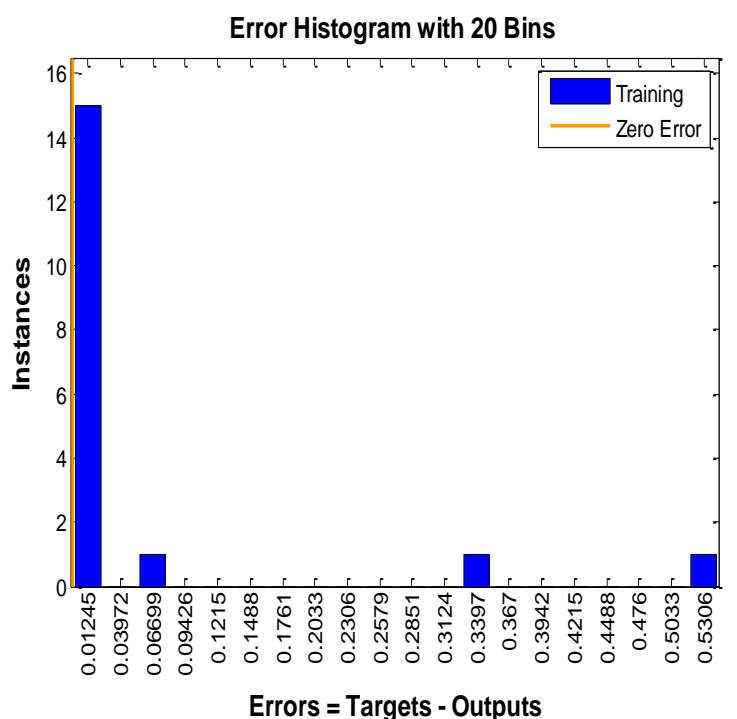

Errors $=$ Targets - Outputs

Fig.8: Error histogram 
Table: 2 Performance of different algorithm for skin diseases classification

\begin{tabular}{|l|l|l|l|l|}
\hline S.N. & Training Algorithms & Epochs & MSE & Gradient \\
\hline & Resilient backpropagation & 347 & $3.5354 \mathrm{e}-07$ & $8.5468 \mathrm{e}-06$ \\
\hline & Scaled conjugate gradient backpropagation & 115 & 0.02269 & $8.6124 \mathrm{e}-07$ \\
\hline & Bayesian regularization backpropagation & 190 & $4.8561 \mathrm{e}-13$ & $1.6337 \mathrm{e}-08$ \\
\hline & Levenberg-Marquardt backpropagation & 105 & $1.0559 \mathrm{e}-10$ & $9.9001 \mathrm{e}-08$ \\
\hline
\end{tabular}

\section{REFERENCES}

[1] Argenziano, Giuseppe, and H. Peter Soyer. "Dermoscopy of pigmented skin lesions-a valuable tool for early." The lancet oncology 2, no. 7 (2001): 443-449.

[2] Vestergaard, M. E., P. H. P. M. Macaskill, P. E. Holt, and S. W. Menzies. "Dermoscopy compared with naked eye examination for the diagnosis of primary melanoma: a meta-analysis of studies performed in a clinical setting." British Journal of Dermatology 159, no. 3 (2008): 669-676.

[3] Ascierto, P. A., G. Palmieri, E. Celentano, R. Parasole, C. Caraco, A. Daponte, M. G. Chiofalo et al. "Sensitivity and specificity of epiluminescence microscopy: evaluation on a sample of 2731 excised cutaneous pigmented lesions." British Journal of Dermatology 142, no. 5 (2000): 893-898.

[4] Stanley, R. Joe, Randy Hays Moss, William Van Stoecker, and Chetna Aggarwal. "A fuzzy-based histogram analysis technique for skin lesion discrimination in dermatology clinical images." Computerized Medical Imaging and Graphics 27, no. 5 (2003): 387-396.

[5] H. Peter Soyer, Harold S. Rabinovitz, and Armand B. Cognetta. "Fuzzy logic techniques for blotch feature evaluation in dermoscopy images." Computerized Medical Imaging and Graphics 33, no. 1 (2009): 50-57.

[6] Xiao, Kong, Lin Danghui, and Shen Lansun. "Segmentation of skin color regions based on fuzzy cluster." In Intelligent Multimedia, Video and Speech Processing, 2004. Proceedings of 2004 International Symposium on, pp. 125-128. IEEE, 2004.

[7] Schmid, Philippe. "Segmentation of digitized dermatoscopic images by two-dimensional color clustering." IEEE Transactions on Medical Imaging 18, no. 2 (1999): 164-171.

[8] Pan, Meisen, Jingtian Tang, and Qi Xiong. "Medical image registration using fuzzy theory." Computer methods in biomechanics and biomedical engineering 15, no. 7 (2012): 721-734.

[9] Bhatt, Rajen B., Gaurav Sharma, Abhinav Dhall, and Santanu Chaudhury. "Efficient skin region segmentation using low complexity fuzzy decision tree model." In India Conference (INDICON), 2009 Annual IEEE, pp. 1-4. IEEE, 2009.
[10] Ma, Zhen, João Manuel RS Tavares, Renato Natal Jorge, and T. Mascarenhas. "A review of algorithms for medical image segmentation and their applications to the female pelvic cavity." Computer Methods in Biomechanics and Biomedical Engineering 13, no. 2 (2010): 235-246.

[11] Chuang, Shao-Hui, Xiaoyan Sun, Wen-Yu Chang, GwoShing Chen, Adam Huang, Jiang Li, and Frederic D. McKenzie. "BCC skin cancer diagnosis based on texture analysis techniques." In SPIE Medical Imaging, pp. 796330-796330. International Society for Optics and Photonics, 2011.

[12] Deng, Yining, and B. S. Manjunath. "Unsupervised segmentation of color-texture regions in images and video." IEEE transactions on pattern analysis and machine intelligence 23, no. 8 (2001): 800-810.

[13] Geman, Stuart, and Donald Geman. "Stochastic relaxation, Gibbs distributions, and the Bayesian restoration of images." IEEE Transactions on pattern analysis and machine intelligence 6 (1984): 721-741.

[14] Vincent, O. R., and Olusegun Folorunso. "A descriptive algorithm for sobel image edge detection." In Proceedings of Informing Science \& IT Education Conference (InSITE), vol. 40, pp. 97-107. 2009.

[15] Urooj, Shabana, and Sudhakar Singh. "A novel computer assisted approach for diagnosis of skin disease." In Computing for Sustainable Global Development (INDIACom), 2015 2nd International Conference on, pp. 1585-1590. IEEE, 2015.

[16] Flusser, Jan, Barbara Zitová, and Toms Suk. "Invariantbased registration of rotated and blurred images." In Geoscience and Remote Sensing Symposium, 1999. IGARSS'99 Proceedings. IEEE 1999 International, vol. 2, pp. 1262-1264. IEEE, 1999.

[17] Chen, Qing, Emil Petriu, and Xiaoli Yang. "A comparative study of Fourier descriptors and Hu's seven moment invariants for image recognition." In Electrical and Computer Engineering, 2004. Canadian Conference on, vol. 1, pp. 103-106. IEEE, 2004.

[18] Ma, Zhen, João Manuel RS Tavares, Renato Natal Jorge, and T. Mascarenhas. "A review of algorithms for medical image segmentation and their applications to the female pelvic cavity." Computer Methods in Biomechanics and Biomedical Engineering 13, no. 2 (2010): 235-246.

[19] Van Leemput, Koen, Frederik Maes, Dirk Vandermeulen, and Paul Suetens. "Automated model- 
International Journal of Computer Applications (0975 - 8887)

Volume 179 - No.31, April 2018

based tissue classification of MR images of the brain." IEEE transactions on medical imaging 18, no. 10 (1999): 897-908.

[20] Manjunath, Bangalore S., and Wei-Ying Ma. "Texture features for browsing and retrieval of image data." IEEE Transactions on pattern analysis and machine intelligence 18, no. 8 (1996): 837-842.

[21] Yang, Jianchao, Kai Yu, Yihong Gong, and Thomas Huang. "Linear spatial pyramid matching using sparse coding for image classification." In Computer Vision and Pattern Recognition, 2009. CVPR 2009. IEEE Conference on, pp. 1794-1801. IEEE, 2009.

[22] Benenson, Rodrigo, Markus Mathias, Radu Timofte, and Luc Van Gool. "Pedestrian detection at 100 frames per second." In Computer Vision and Pattern Recognition (CVPR), 2012 IEEE Conference on, pp. 2903-2910. IEEE, 2012. 